\title{
Existence and positivity of solutions for a totally nonlinear neutral periodic differential equation
}

\author{
Abdelouaheb Ardjouni and Ahcene Djoudi
}




\title{
EXISTENCE AND POSITIVITY OF SOLUTIONS FOR A TOTALLY NONLINEAR NEUTRAL PERIODIC DIFFERENTIAL EQUATION
}

\author{
ABDELOUAHEB ARDJOUNI, AHCENE DJOUDI
}

Received 25 March, 2013

\begin{abstract}
In this paper, we use a modification of Krasnoselskii's fixed point theorem introduced by Burton (see [6] Theorem 3) to establish new results on the existence and positivity of solutions for the totally nonlinear neutral periodic differential equation of the form

$$
\begin{aligned}
\frac{d}{d t} x(t) & =-a(t) x^{3}(t)+\frac{d}{d t} Q(t, x(g(t)))+G\left(t, x^{3}(t), x^{3}(g(t))\right), \\
x(t+T) & =x(t) .
\end{aligned}
$$

We invert this equation to construct a sum of a completely continuous map and a large contraction which is suitable for the application of a modification of Krasnoselskii's theorem.
\end{abstract}

2010 Mathematics Subject Classification: 34A37; 34A12; 39A05

Keywords: fixed point theory, large contraction, totally nonlinear neutral differential equation, periodic

\section{INTRODUCTION}

In the past decades, very much attention has been given to neutral differential equations (see [1-16], [18] and the references therein). The main reason for this interest is that delay differential equations play an important role in applications. For instance, in biological applications, delay equations give better description of fluctuations in population than the ordinary ones. As known in Hale [13], Hale and Lunel [14] neutral delay differential equations appear as models of electrical networks which contain lossless transmission lines. Such networks arise, for example, in high speed computers where lossless transmission lines are used to interconnect switching circuits.

Existence, uniqueness, stability and positivity of solutions of functional differential equations are of great interest in mathematics and its applications to the modeling of various practical problems (see [1-16], [18]). Positivity is one of the most common and most important characteristics of mathematical models. In problem of economics, the positivity is quite important for processes that model interest rate dynamics on financial market, because the interest must be positive. Also, in fluid flow problems, densities, pressures, and concentrations are always positive. 
The primary motivation for the present work are the papers by Y. M. Dib et al. [10] and Yankson [18]. In [10], the authors used Krasnoselskii's fixed point theorem to establish the existence of periodic solutions for the nonlinear neutral differential equation

$$
\frac{d}{d t} x(t)=-a(t) x(t)+\frac{d}{d t} Q(t, x(t-g(t)))+G(t, x(t), x(t-g(t))),
$$

where the nonlinear terms $Q$ and $G$ and the function $a$ are continuous in all arguments. Also, the authors used the contraction mapping principle to show the uniqueness of periodic solutions and stability of the zero solution of (1.1).

In [18], the author used a modification of Krasnoselskii's fixed point theorem to establish the existence and positivity of solutions for the totally nonlinear neutral periodic differential equation

$$
\begin{aligned}
x^{\prime}(t) & =-a(t) x^{3}(t)+c(t) x^{\prime}(g(t)) g^{\prime}(t)+q\left(t, x^{3}(g(t))\right), \\
x(t+T) & =x(t),
\end{aligned}
$$

where $T>0$ be fixed, the nonlinear term $q$ is an $L^{1}$-Carathéodory function and the function $a \in L^{1}[0, T]$ is bounded. Also, the author used the contraction mapping principle to show the uniqueness of periodic solutions of (1.2).

In this paper, we consider the totally nonlinear neutral periodic differential equation

$$
\begin{aligned}
\frac{d}{d t} x(t) & =-a(t) x^{3}(t)+\frac{d}{d t} Q(t, x(g(t)))+G\left(t, x^{3}(t), x^{3}(g(t))\right), \\
x(t+T) & =x(t),
\end{aligned}
$$

where $T>0$ be fixed, the nonlinear terms $Q$ and $G$ are an $L^{1}$-Carathéodory functions and the function $a \in L^{1}[0, T]$ is bounded. Our purpose here is to use a modification of Krasnoselskii's fixed point theorem due Burton (see [6] Theorem 3) to show the existence and positivity of periodic solutions for equation (1.3). Clearly, the present problem is totally nonlinear so that the variation of parameters can not be applied directly. Then, we resort to the idea of adding and subtracting a linear term. As noted by Burton in [6], the added term destroys a contraction already present in part of the equation but it replaces it with the so called a large contraction mapping which is suitable for fixed point theory. During the process we have to transform (1.3) into an integral equation written as a sum of two mappings; one is a large contraction and the other is completely continuous. After that, we use a variant of Krasnoselskii fixed point theorem, to show the existence and positivity of a periodic solution.

Note that in our consideration the neutral term $\frac{d}{d t} Q(t, x(g(t)))$ of (1.3) produces nonlinearity in the derivative term $\frac{d}{d t} x(g(t))$. The neutral term $\frac{d}{d t} x(g(t))$ of (1.2) in [18] enters linearly. As a consequence, our analysis is different form that in [18].

In Section 2, we present the inversion of totally nonlinear neutral periodic differential equation (1.3) and Krasnoselskii-Burton's fixed point theorem. For details 
on Krasnoselskii-Burton theorem we refer the reader to [6,7]. We present our main results on existence and positivity in Section 3.

\section{PRELIMINARIES}

The following definition is essential in our analysis.

Definition 1. A function $F:[0, T] \times \mathbb{R}^{n} \rightarrow \mathbb{R}$ is an $L^{1}$-Carathéodory function if it satisfies the following conditions

(c1) For each $z \in \mathbb{R}^{n}$, the mapping $t \rightarrow F(t, z)$ is Lebesgue measurable.

(c2) For almost all $t \in[0, T]$, the mapping $z \rightarrow F(t, z)$ is continuous on $\mathbb{R}^{n}$.

(c3) For each $r>0$, there exists $f_{r} \in L^{1}([0, T], \mathbb{R})$ such that for almost all $t \in$ $[0, T]$ and for all $z$ such that $|z|<r$, we have $|F(t, z)| \leq f_{r}(t)$.

Define the set $P_{T}=\{\varphi \in C(\mathbb{R}, \mathbb{R}): \varphi(t+T)=\varphi(t)\}$ and the norm $\|\varphi\|=\sup _{0 \leq t \leq T}|\varphi(t)|$. Then $\left(P_{T},\|\|.\right)$ is a Banach space. We will assume that the following conditions hold.

(h1) $a \in L_{L o c}^{1}(\mathbb{R}, \mathbb{R})$ is positive and bounded, satisfies $a(t+T)=a(t)$ for all $t$ and

$$
1-e^{-\int_{t-T}^{t} a(s) d s} \equiv \frac{1}{\eta} \neq 0 .
$$

(h2) $g \in P_{T}$.

(h3) $Q$ and $G$ are an $L^{1}$-Carathéodory functions, and $Q(t+T, x)=Q(t, x)$, $G(t+T, x, y)=Q(t, x, y)$ for all $t$.

Lemma 1. Suppose that conditions $(h 1),(h 2)$ and $(h 3)$ hold. Then $x \in P_{T}$ is a solution of equation (1.3) if and only if

$$
\begin{aligned}
x(t) & =\eta \int_{t-T}^{t} a(u)\left[x(u)-x^{3}(u)\right] e^{-\int_{u}^{t} a(s) d s} d u+Q(t, x(g(t))) \\
& +\eta \int_{t-T}^{t}\left[G\left(u, x^{3}(u), x^{3}(g(u))\right)-a(u) Q(u, x(g(u)))\right] e^{-\int_{u}^{t} a(s) d s} d u .
\end{aligned}
$$

Proof. Let $x \in P_{T}$ be a solution of (1.3). First we write this equation as

$$
\begin{gathered}
\frac{d}{d t}\{x(t)-Q(t, x(g(t)))\} \\
=-a(t)\{x(t)-Q(t, x(g(t)))\}+a(t)\left\{x(t)-x^{3}(t)\right\} \\
+G\left(t, x^{3}(t), x^{3}(g(t))\right)-a(t) Q(t, x(g(t))) .
\end{gathered}
$$

Multiply both sides of the above equation by $e^{\int_{0}^{t} a(s) d s}$ and then integrate from $t-T$ to $t$ to obtain

$$
\int_{t-T}^{t}\left[(x(u)-Q(u, x(g(u)))) e^{\int_{0}^{u} a(s) d s}\right]^{\prime} d u
$$




$$
\begin{aligned}
& =\int_{t-T}^{t} a(u)\left[x(u)-x^{3}(u)\right] e^{\int_{0}^{u} a(s) d s} d u \\
& +\int_{t-T}^{t}\left[G\left(u, x^{3}(u), x^{3}(g(u))\right)-a(u) Q(u, x(g(u)))\right] e^{\int_{0}^{u} a(s) d s} d u .
\end{aligned}
$$

As a consequence, we arrive

$$
\begin{aligned}
& (x(t)-Q(t, x(g(t)))) e^{\int_{0}^{t} a(s) d s} \\
& -(x(t-T)-Q(t-T, x(g(t-T)))) e^{\int_{0}^{t-T} a(s) d s} \\
& =\int_{t-T}^{t} a(u)\left[x(u)-x^{3}(u)\right] e^{\int_{0}^{u} a(s) d s} d u \\
& +\int_{t-T}^{t}\left[G\left(u, x^{3}(u), x^{3}(g(u))\right)-a(u) Q(u, x(g(u)))\right] e^{\int_{0}^{u} a(s) d s} d u .
\end{aligned}
$$

Dividing both sides of the above equation by $e^{\int_{0}^{t} a(s) d s}$ and the fact that $x(t)=$ $x(t-T), g(t)=g(t-T)$ and $Q(t, x)=Q(t-T, x)$, we obtain

$$
\begin{aligned}
& x(t)-Q(t, x(g(t))) \\
& =\eta \int_{t-T}^{t} a(u)\left[x(u)-x^{3}(u)\right] e^{-\int_{u}^{t} a(s) d s} d u \\
& +\eta \int_{t-T}^{t}\left[G\left(u, x^{3}(u), x^{3}(g(u))\right)-a(u) Q(u, x(g(u)))\right] e^{-\int_{u}^{t} a(s) d s} d u .
\end{aligned}
$$

Since each step is reversible, the converse follows easily. This completes the proof.

Krasnoselskii (see [5, 17]) combined the contraction mapping theorem and Shauder's theorem and formulated the following hybrid and attractive result.

Theorem 1 (Krasnoselskii). Let $\mathrm{M}$ be a closed convex nonempty subset of a $\mathrm{Ba}$ nach space $(S,\|\|$.$) . Suppose that A$ and $B$ map $\mathbb{M}$ into $S$ such that

(i) $x, y \in \mathbb{M}$, implies $A x+B y \in \mathbb{M}$,

(ii) $A$ is completely continuous, and

(i i i) $B$ is a contraction with constant $\alpha<1$.

Then there exists $z \in \mathbb{M}$ with $z=A z+B z$.

This is a captivating result and has a number of interesting applications. In recent years much attention has paid to this theorem. Burton [5] observed that Krasnoselskii result can be more interesting in applications with certain changes and formulated in Theorem 3 bellow (see [4] for the proof).

Definition 2 (Large Contraction). Let $(M, d)$ be a metric space and $B: \mathbb{M} \rightarrow \mathbb{M}$. $B$ is said to be a large contraction if $\varphi, \psi \in \mathbb{M}$, with $\varphi \neq \psi$ then $d(B \varphi, B \psi)<$ 
$d(\varphi, \psi)$ and if for all $\varepsilon>0$ there exists $\delta<1$ such that

$$
[\varphi, \psi \in \mathbb{M}, d(\varphi, \psi) \geq \varepsilon] \Rightarrow d(B \varphi, B \psi) \leq \delta d(\varphi, \psi) .
$$

Theorem 2. Let $(\mathbb{M}, d)$ be a complete metric space and $B$ be a large contraction. Suppose there is an $x \in \mathbb{M}$ and $L>0$, such that $d\left(x, B^{n} x\right) \leq L$ for all $n \geq 1$. Then $B$ has a unique fixed point in $\mathrm{M}$.

Theorem 3 (Krasonselskii-Burton). Let $\mathrm{M}$ be a closed bounded convex non-empty subset of a Banach space $(S,\|\|$.$) . Suppose that A, B$ map $\mathbb{M}$ into $\mathbb{M}$ and that

(i) $\forall x, y \in \mathbb{M} \Rightarrow A x+B y \in \mathbb{M}$,

(ii) $A$ is completely continuous, and

(ii i) $B$ is a large contraction.

Then there is a $z \in \mathbb{M}$ with $z=A z+B z$.

\section{EXISTENCE RESULTS}

We present or existence results in this section. To this end, we first define the operator $H$ by

$$
\begin{gathered}
(H \varphi)(t)=\eta \int_{t-T}^{t} a(u)\left[\varphi(u)-\varphi^{3}(u)\right] e^{-\int_{u}^{t} a(s) d s} d u+Q(t, \varphi(g(t))) \\
+\eta \int_{t-T}^{t}\left[G\left(u, \varphi^{3}(u), \varphi^{3}(g(u))\right)-a(u) Q(u, \varphi(g(u)))\right] e^{-\int_{u}^{t} a(s) d s} d u,
\end{gathered}
$$

From Lemma 1 we see that fixed points of $H$ are solutions of (1.3) and vice versa. In order to employ theorem 3 we need to express the operator $H$ as the sum of two operators, one of which is completely continuous and the other of which is a large contraction. Let $(H \varphi)(t)=(A \varphi)(t)+(B \varphi)(t)$ where

$$
\begin{gathered}
(A \varphi)(t)=Q(t, \varphi(g(t))) \\
+\eta \int_{t-T}^{t}\left[G\left(u, \varphi^{3}(u), \varphi^{3}(g(u))\right)-a(u) Q(u, \varphi(g(u)))\right] e^{-\int_{u}^{t} a(s) d s} d u,
\end{gathered}
$$

and

$$
(B \varphi)(t)=\eta \int_{t-T}^{t} a(u)\left[\varphi(u)-\varphi^{3}(u)\right] e^{-\int_{u}^{t} a(s) d s} d u .
$$

We need the following condition on the nonlinear term $Q$.

(h4) The function $Q(t, x)$ is continuous in $t$ and there exist bounded positive periodic functions $q_{1}, q_{2} \in L^{1}[0, T]$, with period $T$, such that

$$
|Q(t, x)| \leq q_{1}(t)|x|+q_{2}(t),
$$

for all $x \in \mathbb{R}$.

Our first lemma in this section shows that $A: P_{T} \rightarrow P_{T}$ is completely continuous.

Lemma 2. Suppose that conditions $(h 1)-(h 4)$ hold. Then $A: P_{T} \rightarrow P_{T}$ is completely continuous. 
Proof. From (3.2) and conditions $(h 1)-(h 3)$, it follows trivially that $e^{-\int_{u+T}^{t+T} a(s) d s}=e^{-\int_{u}^{t} a(s) d s}$. Consequently, we have that

$$
(A \varphi)(t+T)=(A \varphi)(t) .
$$

That is, if $\varphi \in P_{T}$ then $A \varphi$ is periodic with period $T$.

To see that $A$ is continuous. Let $\left\{\varphi_{i}\right\} \subset P_{T}$ be such that $\varphi_{i} \rightarrow \varphi$ as $i \rightarrow \infty$. By the Dominated Convergence Theorem,

$$
\begin{aligned}
& \lim _{i \rightarrow \infty}\left|\left(A \varphi_{i}\right)(t)-(A \varphi)(t)\right| \\
& \leq \lim _{i \rightarrow \infty}\left|Q\left(t, \varphi_{i}(g(t))\right)-Q(t, \varphi(g(t)))\right| \\
& \lim _{i \rightarrow \infty} \eta \int_{t-T}^{t}\left\{\left|G\left(u, \varphi_{i}^{3}(u), \varphi_{i}^{3}(g(u))\right)-G\left(u, \varphi^{3}(u), \varphi^{3}(g(u))\right)\right|\right. \\
& \left.+a(u)\left|Q\left(u, \varphi_{i}(g(u))\right)-Q(u, \varphi(g(u)))\right|\right\} e^{-\int_{u}^{t} a(s) d s} d u \\
& =\lim _{i \rightarrow \infty}\left|Q\left(t, \varphi_{i}(g(t))\right)-Q(t, \varphi(g(t)))\right| \\
& +\eta \int_{t-T}^{t}\left\{\lim _{i \rightarrow \infty}\left|G\left(u, \varphi_{i}^{3}(u), \varphi_{i}^{3}(g(u))\right)-G\left(u, \varphi^{3}(u), \varphi^{3}(g(u))\right)\right|\right. \\
& \left.+a(u) \lim _{i \rightarrow \infty}\left|Q\left(u, \varphi_{i}(g(u))\right)-Q(u, \varphi(g(u)))\right|\right\} e^{-\int_{u}^{t} a(s) d s} d u \\
& =0 .
\end{aligned}
$$

Hence $A: P_{T} \rightarrow P_{T}$ is continuous.

Finally, we show that $A$ is completely continuous. Let $\mathfrak{C} \subset P_{T}$ be a closed bounded subset and let $C$ be such that $\|\varphi\| \leq C$ for all $\varphi \in \mathfrak{C}$. then

$$
\begin{gathered}
|(A \varphi)(t)| \leq q_{1}^{*} C+q_{2}^{*} \\
+\eta \int_{t-T}^{t}\left[\left|G\left(u, \varphi^{3}(u), \varphi^{3}(g(u))\right)\right|+a(u)|Q(u, \varphi(g(u)))|\right] e^{-\int_{u}^{t} a(s) d s} d u \\
\leq q_{1}^{*} C+q_{2}^{*}+\eta N\left\{\int_{t-T}^{t} g_{C}(u) d u+\int_{t-T}^{t} a(u) q_{C}(u) d u\right\} \\
\leq q_{1}^{*} C+q_{2}^{*}+\eta N\left\{\int_{t-T}^{t} g_{C}(u) d u+\alpha \int_{t-T}^{t} q_{C}(u) d u\right\} \equiv K,
\end{gathered}
$$

where $q_{1}^{*}=\max _{u \in[0, T]} q_{1}(t), q_{2}^{*}=\max _{u \in[0, T]} q_{2}(t), N=\max _{u \in[t-T, t]} e^{-\int_{u}^{t} a(s) d s}$ and $\alpha=$ $\max _{u \in[t-T, t]} a(u)$. And so, the family of functions $A \varphi$ is uniformly bounded.

Again, let $\varphi \in \mathfrak{C}$. Without loss of generality, we can pick $t_{1}<t_{2}$ such that $t_{2}-t_{1}<$ $T$. Then

$$
\left|(A \varphi)\left(t_{2}\right)-(A \varphi)\left(t_{1}\right)\right|
$$




$$
\begin{gathered}
=\mid Q\left(t_{2}, \varphi\left(t_{2}\right)\right) \\
+\eta \int_{t_{2}-T}^{t_{2}}\left[G\left(u, \varphi^{3}(u), \varphi^{3}(g(u))\right)-a(u) Q(u, \varphi(g(u)))\right] e^{-\int_{u}^{t_{2}} a(s) d s} d u \\
-Q\left(t_{1}, \varphi\left(t_{1}\right)\right) \\
-\eta \int_{t_{1}-T}^{t_{1}}\left[G\left(u, \varphi^{3}(u), \varphi^{3}(g(u))\right)-a(u) Q(u, \varphi(g(u)))\right] e^{-\int_{u}^{t_{1}} a(s) d s} d u \mid .
\end{gathered}
$$

We can rewrite the left hand side as the sum of three integrals. We obtain the following

$$
\begin{aligned}
& \left|(A \varphi)\left(t_{2}\right)-(A \varphi)\left(t_{1}\right)\right| \\
& \leq\left|Q\left(t_{2}, \varphi\left(t_{2}\right)\right)-Q\left(t_{1}, \varphi\left(t_{1}\right)\right)\right| \\
& +\eta \int_{t_{1}}^{t_{2}}\left[\left|G\left(u, \varphi^{3}(u), \varphi^{3}(g(u))\right)\right|+a(u)|Q(u, \varphi(g(u)))|\right] e^{-\int_{u}^{t_{2}} a(s) d s} d u \\
& +\eta \int_{t_{2}-T}^{t_{1}}\left[\left|G\left(u, \varphi^{3}(u), \varphi^{3}(g(u))\right)\right|+a(u)|Q(u, \varphi(g(u)))|\right] \\
& \times\left|e^{-\int_{u}^{t_{2}} a(s) d s}-e^{-\int_{u}^{t_{1}} a(s) d s}\right| d u \\
& +\eta \int_{t_{1}-T}^{t_{2}-T}\left[\left|G\left(u, \varphi^{3}(u), \varphi^{3}(g(u))\right)\right|+a(u)|Q(u, \varphi(g(u)))|\right] e^{-\int_{u}^{t_{1}} a(s) d s} d u \\
& \leq\left|Q\left(t_{2}, \varphi\left(t_{2}\right)\right)-Q\left(t_{1}, \varphi\left(t_{1}\right)\right)\right|+2 \eta N\left\{\int_{t_{1}}^{t_{2}} g_{C}(u)+\alpha q_{C}(u) d u\right\} \\
& +\eta \int_{t_{2}-T}^{t_{1}}\left[g_{C}(u)+\alpha q_{C}(u)\right]\left|e^{-\int_{u}^{t_{2}} a(s) d s}-e^{-\int_{u}^{t_{1}} a(s) d s}\right| d u .
\end{aligned}
$$

Now $\left|Q\left(t_{2}, \varphi\left(t_{2}\right)\right)-Q\left(t_{1}, \varphi\left(t_{1}\right)\right)\right| \rightarrow 0$ and $\int_{t_{1}}^{t_{2}} g_{C}(u)+\alpha q_{C}(u) d u \rightarrow 0$ as $\left(t_{2}-t_{1}\right) \rightarrow 0$. Also, since

$$
\begin{aligned}
& \int_{t_{2}-T}^{t_{1}}\left[g_{C}(u)+\alpha q_{C}(u)\right]\left|e^{-\int_{u}^{t_{2}} a(s) d s}-e^{-\int_{u}^{t_{1}} a(s) d s}\right| d u \\
& \leq \int_{0}^{T}\left[g_{C}(u)+\alpha q_{C}(u)\right]\left|e^{-\int_{u}^{t_{2}} a(s) d s}-e^{-\int_{u}^{t_{1}} a(s) d s}\right| d u,
\end{aligned}
$$

and $\left|e^{-\int_{u}^{t_{2}} a(s) d s}-e^{-\int_{u}^{t_{1}} a(s) d s}\right| \rightarrow 0$ as $\left(t_{2}-t_{1}\right) \rightarrow 0$, then by the Dominated Convergence Theorem,

$$
\int_{t_{2}-T}^{t_{1}}\left[g_{C}(u)+\alpha q_{C}(u)\right]\left|e^{-\int_{u}^{t_{2}} a(s) d s}-e^{-\int_{u}^{t_{1}} a(s) d s}\right| d u \rightarrow 0,
$$


as $\left(t_{2}-t_{1}\right) \rightarrow 0$. Thus $\left|A \varphi\left(t_{2}\right)-A \varphi\left(t_{1}\right)\right| \rightarrow 0$ as $\left(t_{2}-t_{1}\right) \rightarrow 0$ independently of $\varphi \in \mathfrak{C}$. As such, the family of functions $A \varphi$ is equicontinuous on $\mathfrak{C}$. By the ArzelàAscoli Theorem, $A$ is completely continuous and the proof is complete.

Proposition 1. Let $\|\cdot\|$ be the supremum norm,

$$
\mathrm{M}=\{\varphi \in S:\|\varphi\| \leq \sqrt{3} / 3\}
$$

and define $(\mathfrak{B} \varphi)(t):=\varphi(t)-\varphi^{3}(t)$. Then $\mathfrak{B}$ is a large contraction of the set $\mathbb{M}$.

Proof. For each $t \in \mathbb{R}$ we have, for $\varphi, \psi$ real functions,

$$
\begin{aligned}
|(\mathfrak{B} \varphi)(t)-(\mathfrak{B} \psi)(t)| & =\left|\varphi(t)-\varphi^{3}(t)-\psi(t)+\psi^{3}(t)\right| \\
& =|\varphi(t)-\psi(t)|\left|1-\left(\varphi^{2}(t)+\varphi(t) \psi(t)+\psi^{2}(t)\right)\right| .
\end{aligned}
$$

Then for

$$
|\varphi(t)-\psi(t)|^{2}=\varphi^{2}(t)-2 \varphi(t) \psi(t)+\psi^{2}(t) \leq 2\left(\varphi^{2}(t)+\psi^{2}(t)\right),
$$

and for $\varphi^{2}(t)+\psi^{2}(t)<1$, we have

$$
\begin{aligned}
|(\mathfrak{B} \varphi)(t)-(\mathfrak{B} \psi)(t)| & =|\varphi(t)-\psi(t)|\left[1-\left(\varphi^{2}(t)+\psi^{2}(t)\right)+|\varphi(t) \psi(t)|\right] \\
& \leq|\varphi(t)-\psi(t)|\left[1-\left(\varphi^{2}(t)+\psi^{2}(t)\right)+\frac{\varphi^{2}(t)+\psi^{2}(t)}{2}\right] \\
& \leq|\varphi(t)-\psi(t)|\left[1-\frac{\varphi^{2}(t)+\psi^{2}(t)}{2}\right] .
\end{aligned}
$$

Thus, $\mathfrak{B}$ is pointwise a large contraction. But application $\mathfrak{B}$ is still a large contraction for the supremum norm. For, let $\varepsilon \in(0,1)$ be given and let $\varphi, \psi \in \mathbb{M}$ with $\|\varphi-\psi\| \geq$ $\varepsilon$.

(a) Suppose that for some $t$ we have $\varepsilon / 2 \leq|\varphi(t)-\psi(t)|$. Then

$$
(\varepsilon / 2)^{2} \leq|\varphi(t)-\psi(t)|^{2} \leq 2\left(\varphi^{2}(t)+\psi^{2}(t)\right)
$$

that is

For all such $t$ we have

$$
\varphi^{2}(t)+\psi^{2}(t) \geq \varepsilon^{2} / 8
$$

$$
|(\mathfrak{B} \varphi)(t)-(\mathfrak{B} \psi)(t)| \leq|\varphi(t)-\psi(t)|\left[1-\frac{\varepsilon^{2}}{16}\right] \leq\|\varphi-\psi\|\left[1-\frac{\varepsilon^{2}}{16}\right] .
$$

(b) Suppose that for some $t$ we have

$$
|\varphi(t)-\psi(t)| \leq \varepsilon / 2
$$

Then

$$
|(\mathfrak{B} \varphi)(t)-(\mathfrak{B} \psi)(t)| \leq|\varphi(t)-\psi(t)| \leq(1 / 2)\|\varphi-\psi\| .
$$

Consequently, we obtain

$$
\|\mathfrak{B} \varphi-\mathfrak{B} \psi\| \leq \max \left[1 / 2,1-\frac{\varepsilon^{2}}{16}\right]\|\varphi-\psi\|
$$




$$
\leq\left(1-\frac{\varepsilon^{2}}{16}\right)\|\varphi-\psi\|
$$

For the rest of the paper we define

$$
\mathbb{M}=\{\varphi \in S:\|\varphi\| \leq L\},
$$

where $L=\sqrt{3} / 3$.

We need the following condition on the nonlinear term $G$.

(h5) There exist positive periodic functions $g_{1}, g_{2}, g_{3} \in L^{1}[0, T]$, with period $T$, such that

$$
|G(t, x, y)| \leq g_{1}(t)|x|+g_{2}(t)|y|+g_{3}(t),
$$

for all $x, y \in \mathbb{R}$.

Lemma 3. Suppose that conditions $(h 4)$ and $(h 5)$ hold. Also suppose there exist constants $\beta, \delta>0$ and $J \geq 3$ such that

$$
\begin{aligned}
{\left[g_{1}(t)+g_{2}(t)\right] L^{3}+g_{3}(t) } & \leq \beta L a(t), \\
q_{1}(t) L+q_{2}(t) & \leq \delta L,
\end{aligned}
$$

and

$$
J(2 \delta+\beta) \leq 1 .
$$

For $A$ defined by (3.3), if $\varphi \in \mathbb{M}$, then $|(A \varphi)(t)| \leq L / J<L$ for all $t$.

Proof. Let $\varphi \in \mathbb{M}$. Then $\|\varphi\| \leq L$. Thus for $A$ defined by (3.2) we have that

$$
\begin{aligned}
|(A \varphi)(t)| & \leq|Q(t, \varphi(g(t)))|+\eta \int_{t-T}^{t}\left|G\left(u, \varphi^{3}(u), \varphi^{3}(g(u))\right)\right| e^{-\int_{u}^{t} a(s) d s} d u \\
& +\eta \int_{t-T}^{t} a(u)|Q(u, \varphi(g(u)))| e^{-\int_{u}^{t} a(s) d s} d u .
\end{aligned}
$$

It follows from conditions $(h 4),(h 5),(3.4)-(3.6)$ that

$$
\begin{aligned}
|(A \varphi)(t)| & \leq q_{1}(t) L+q_{2}(t) \\
& +\eta \int_{t-T}^{t}\left\{\left[g_{1}(u)+g_{2}(u)\right] L^{3}+g_{3}(u)\right\} e^{-\int_{u}^{t} a(s) d s} d u \\
& +\eta \int_{t-T}^{t} a(u)\left\{q_{1}(u) L+q_{2}(u)\right\} e^{-\int_{u}^{t} a(s) d s} d u \\
& \leq \delta L+\beta L \eta \int_{t-T}^{t} a(u) e^{-\int_{u}^{t} a(s) d s} d u+\delta L \eta \int_{t-T}^{t} a(u) e^{-\int_{u}^{t} a(s) d s} d u \\
& \leq(2 \delta+\beta) L \leq \frac{L}{J}<L .
\end{aligned}
$$

Therefore $A$ maps $M$ into itself. 
Lemma 4. Suppose $(h 1)-(h 5)$ and (3.4)-(3.6) hold. For A, B defined in (3.2) and (3.3), if $\varphi, \psi \in \mathrm{M}$ are arbitrary, then

$$
A \varphi+B \psi: \mathrm{M} \rightarrow \mathrm{M} .
$$

Moreover, $B$ is a large contraction on $\mathrm{M}$ with a unique fixed point in $\mathrm{M}$.

Proof. Let $\varphi, \psi \in M$ be arbitrary. Note first that $|\psi(t)| \leq \sqrt{3} / 3$ implies

$$
\left|\psi(t)-\psi^{3}(t)\right| \leq(2 \sqrt{3}) / 9 .
$$

Using the definition of $B$, and the result of Lemma 4 , we obtain

$$
\begin{aligned}
|(A \varphi)(t)+(B \psi)(t)| & \leq|(A \varphi)(t)|+|(B \psi)(t)| \\
& \leq \frac{\sqrt{3}}{3 J}+\frac{2 \sqrt{3}}{9} \leq L .
\end{aligned}
$$

Thus, $A \varphi+B \psi \in \mathrm{M}$.

Left to show that $B$ is a large contraction with a unique fixed point in M. Proposition 1 shows that $\psi-\psi^{3}$ is a large contraction in the supremum norm. For any $\varepsilon$, from the proof of that proposition, we have found a $\lambda<1$, such that

$$
\begin{aligned}
|(B \psi)(t)-(B \varphi)(t)| & \leq \eta \int_{t-T}^{t} a(u) \lambda\|\psi-\varphi\| e^{-\int_{u}^{t} a(s) d s} d u \\
& \leq \lambda\|\psi-\varphi\| .
\end{aligned}
$$

Further, since $0 \in \mathbb{M}$ the above inequality shows, when $\varphi=0$, we see that $B: M \rightarrow$ $M$. This completes the proof.

Theorem 4. Let $(S,\|\|$.$) be the Banach space of continuous T-periodic real func-$ tions and $\mathrm{M}=\{\varphi \in S:\|\varphi\| \leq L\}$, where $L=\sqrt{3} / 3$. Suppose $(h 1)-(h 5)$ and (3.4)-(3.6) hold. Then equation (1.3) possesses a T-periodic solution $\varphi$ in the subset M.

Proof. By Lemma 1, $\varphi$ is a solution of (1.3) if

$$
\varphi=A \varphi+B \varphi,
$$

where $A$ and $B$ are given by (3.2), (3.3) respectively. By Lemma 2, $A: \mathbb{M} \rightarrow \mathbb{M}$ is completely continuous. By Lemma $4, A \varphi+B \psi \in \mathbb{M}$ whenever $\varphi, \psi \in \mathbb{M}$. Moreover, $B: M \rightarrow \mathbb{M}$ is a large contraction. Clearly, all the hypotheses of Theorem 3 of Krasnoselskii-Burton are satisfied. Thus, there exists a fixed point $\varphi \in \mathbb{M}$ such that $\varphi=A \varphi+B \varphi$. Hence (1.3) has a $T$-periodic solution in $\mathbf{M}$.

For our last result, we give sufficient conditions under which there exists positive solutions of equation (1.3). We begin by defining some new quantities. Let

$$
m=\min _{u \in[t-T, t]} e^{-\int_{u}^{t} a(s) d s}, M=\max _{u \in[t-T, t]} e^{-\int_{u}^{t} a(s) d s} .
$$


Given constants $0<\mathfrak{L}<\mathfrak{K} \leq \sqrt{3} / 3$, define the set

$\mathbb{M}_{1}=\left\{\varphi \in P_{T}: \mathfrak{L} \leq \varphi(t) \leq \mathfrak{K}, t \in[0, T]\right\}$.

Assume that the following conditions hold.

(h6) There exist constants $0<q^{*}<L^{*}$ such that $q^{*} \mathfrak{L} \leq Q(t, \rho) \leq L^{*} \mathfrak{K}$ for all $\rho \in \mathbb{M}_{1}$ and $t \in[0, T]$.

(h7) There exist constants $0<\mathfrak{L}<\mathfrak{K}$ such that

$$
\frac{\left(1-q^{*}\right) \mathfrak{L}}{\eta m T} \leq a(u)\left[\sigma-\sigma^{3}\right]+G\left(u, \sigma^{3}, \rho^{3}\right)-a(u) Q(u, \rho) \leq \frac{\left(1-L^{*}\right) \mathfrak{K}}{\eta M T},
$$

for all $\sigma, \rho \in \mathrm{M}_{1}$ and $u \in[t-T, t]$.

Theorem 5. Suppose that conditions $(h 1)-(h 4),(h 6)$ and $(h 7)$ hold. Then there exists a positive solution of (1.3).

Proof. As in the proof of Theorem 4, we just need to show that condition (i ii i) of Theorem 3 is satisfied. Let $\varphi, \psi \in \mathbb{M}_{1}$. Then

$$
\begin{aligned}
& (A \varphi)(t)+(B \psi)(t) \\
& =Q(t, \psi(g(t)))+\eta \int_{t-T}^{t}\left\{a(u)\left[\varphi(u)-\varphi^{3}(u)\right]\right. \\
& \left.+G\left(u, \varphi^{3}(u), \varphi^{3}(g(u))\right)-a(u) Q(u, \varphi(g(u)))\right\} e^{-\int_{u}^{t} a(s) d s} d u \\
& \geq q^{*} \mathfrak{L}+\eta m T \frac{\left(1-q^{*}\right) \mathfrak{L}}{\eta m T}=\mathfrak{L} .
\end{aligned}
$$

Likewise

$$
(A \varphi)(t)+(B \psi)(t) \leq L^{*} \mathfrak{K}+\eta M T \frac{\left(1-L^{*}\right) \mathfrak{K}}{\eta M T}=\mathfrak{K} .
$$

By Theorem 3, the operator $H$ has a fixed point in $M_{1}$. This fixed point is a positive solution of (1.3) and the proof is complete.

\section{REFERENCES}

[1] A. Ardjouni and A. Djoudi, "Existence of periodic solutions for a second order nonlinear neutral differential equation with functional delay," Electronic Journal of Qualitative Theory of Differential Equation, vol. 2012, no. 31, pp. 1-9, 2012.

[2] A. Ardjouni and A. Djoudi, "Existence of periodic solutions for totally nonlinear neutral differential equations with variable delay," Sarajevo J. Math., vol. 8, no. 1, pp. 107-117, 2012.

[3] A. Ardjouni and A. Djoudi, "Existence of positive periodic solutions for a nonlinear neutral differential equation with variable delay," Appl. Math. E-Notes, vol. 12, pp. 94-101, 2012.

[4] T. A. Burton, "Integral equations, implicit functions, and fixed points," Proc. Am. Math. Soc., vol. 124, no. 8, pp. 2383-2390, 1996.

[5] T. A. Burton, "A fixed-point theorem of Krasnoselskii," Appl. Math. Lett., vol. 11, no. 1, pp. 85-88, 1998.

[6] T. A. Burton, "Liapunov functionals, fixed points, and stability by Krasnoselskii's theorem," Nonlinear Stud., vol. 9, no. 2, pp. 181-190, 2002. 
[7] T. A. Burton, Stability by fixed point theory for functional differential equations. Mineola: Dover Publications, 2006.

[8] F. Chen, "Positive periodic solutions of neutral Lotka-Volterra system with feedback control," Appl. Math. Comput., vol. 162, no. 3, pp. 1279-1302, 2005.

[9] F. Chen and J. Shi, "Periodicity in a nonlinear predator-prey system with state dependent delays," Acta Math. Appl. Sin., Engl. Ser., vol. 21, no. 1, pp. 49-60, 2005.

[10] Y. M. Dib, M. R. Maroun, and Y. N. Raffoul, "Periodicity and stability in neutral nonlinear differential equations with functional delay." Electron. J. Differ. Equ., vol. 2005, p. 11, 2005.

[11] M. Fan, K. Wang, P. J. Y. Wong, and R. P. Agarwal, "Periodicity and stability in periodic $n$-species Lotka-Volterra competition system with feedback controls and deviating arguments," Acta Math. Sin., Engl. Ser., vol. 19, no. 4, pp. 801-822, 2003.

[12] D. Hafsia and D. Ahcéne, "Existence of periodic solutions for neutral nonlinear differential equations with variable delay," Electron. J. Differ. Equ., vol. 2010, p. 8, 2010.

[13] J. Hale, Theory of functional differential equations. 2nd ed., ser. Applied Mathematical Sciences. New York - Heidelberg - Berlin: Springer-Verlag, 1977, vol. 3.

[14] J. K. Hale and S. M. Verduyn Lunel, Introduction to functional differential equations, ser. Applied Mathematical Sciences. New York: Springer-Verlag, 1993, vol. 99.

[15] E. R. Kaufmann, "A nonlinear neutral periodic differential equation," Electron. J. Differ. Equ., vol. 2010, p. 8, 2010.

[16] Y. Raffoul, "Positive periodic solutions in neutral nonlinear differential equations," Electron. $J$. Qual. Theory Differ. Equ., vol. 2007, p. 10, 2007.

[17] D. R. Smart, Fixed point theorems., ser. Cambridge Tracts in Mathematics. London: Cambridge University Press, 1974, vol. 66.

[18] E. Yankson, "Existence and positivity of solutions for a nonlinear periodic differential equation," Arch. Math., Brno, vol. 48, no. 4, pp. 261-270, 2012.

\section{Author's address}

Abdelouaheb Ardjouni, Ahcene Djoudi

Abdelouaheb Ardjouni, Ahcene Djoudi, Department of Mathematics, Faculty of Sciences, University of Annaba, P.O. Box 12 Annaba, Algeria

E-mail address: abd_ardjouni@yahoo.fr, adjoudi@yahoo.com 